\title{
Predictive value of peripheral lymphocyte counts for immune checkpoint inhibitor efficacy in advanced head and neck squamous cell carcinoma
}

\author{
JONG CHUL PARK ${ }^{1,2}$, JULIA DURBECK ${ }^{3}$ and JOHN R. CLARK ${ }^{1,2}$ \\ ${ }^{1}$ Department of Medicine, Massachusetts General Hospital Cancer Center, Boston, MA 02114; ${ }^{2}$ Harvard Medical School, \\ Boston, MA 02115; ${ }^{3}$ Department of Otolaryngology, Massachusetts Eye and Ear, Boston, MA 02114, USA
}

Received June 20, 2020; Accepted September 9, 2020

DOI: $10.3892 / \mathrm{mco} .2020 .2157$

\begin{abstract}
Anti-programmed death 1 (PD-1) immune checkpoint inhibitors (ICI) have revolutionized the treatment of advanced head and neck squamous cell carcinoma (HNSCC) but benefit only a small subset of patients. Several studies have previously assessed the predictive value of peripheral lymphocyte count for ICI therapy responses; however the optimal lymphocyte measure for the best predictive value in HNSCC is unknown. The present study examined the predictive values of multiple peripheral lymphocyte measures for anti-PD-1 ICI therapy in advanced HNSCC. Clinicopathologic data were retrospectively collected on patients with recurrent or metastatic HNSCC who had received anti-PD-1 therapy. The association between clinical outcomes and various peripheral lymphocyte count measures was analyzed, including absolute lymphocyte count (ALC) and neutrophil-to-lymphocyte ratios (NLR) at baseline, week 6, and change from baseline to week 6 . The primary outcome of interest was progression-free survival (PFS). A total of 108 patients with HNSCC who had received anti-PD-1 therapy were identified. The median PFS was 4.1 months. Week 6 high ALC $(\geq 0.77)$ and low NLR $(<6.2)$ were associated with a longer PFS (5.6 vs. 3.1 months, $\mathrm{P}=0.002$; and 8.7 vs. 2.9 months, $\mathrm{P}=0.001$, respectively). Decreased NLR during treatment was also associated with an improved PFS (6.7 vs. 2.7 months; $\mathrm{P}=0.015$ ).
\end{abstract}

Correspondence to: Dr Jong Chul Park, Department of Medicine, Massachusetts General Hospital Cancer Center, 55 Fruit St, Boston, MA 02114, USA

E-mail: jpark73@mgh.harvard.edu

Abbreviations: ALC, absolute lymphocyte count; ANC, absolute neutrophil count; CI, confidence interval; ECOG, Eastern Cooperative Oncology Group; HNSCC, head and neck squamous cell carcinoma; HPV, human papilloma virus; HR, hazard ratio; ICI, immune checkpoint inhibitor; NLR, neutrophil-to-lymphocyte ratio; PD-1, programmed cell death 1; PD-L1, programmed death-ligand 1; PFS, progression-free survival; OS, overall survival; PY, pack year

Key words: absolute lymphocyte count, neutrophil-to-lymphocyte ratio, immune checkpoint inhibitors, head and neck cancer
Baseline lymphocyte counts and absolute lymphocyte changes during treatment did not predict ICI outcome. The present single institution retrospective study suggested that ALC and NLR values at week 6 , and on-treatment NLR dynamic change have predictive value for anti-PD-1 therapy response.

\section{Introduction}

Introduction of immunotherapy with immune checkpoint inhibitors (ICI) has changed the therapeutic landscape of advanced head and neck squamous cell carcinoma (HNSCC), but only a small subset of patients with HNSCC benefit from ICI, with an overall response rate of $13-17 \%$ as a monotherapy (1-3). Programmed death-ligand 1 (PD-L1) expression is currently used as a predictive biomarker to select patients for anti-PD1 inhibitory therapy. However, PD-L1 expression is a dynamic biomarker with significant intratumoral heterogeneity and has a low predictive value overall. Additionally, obtaining tissue for PD-L1 testing is not always feasible. Identification of a reliable and accessible clinical marker for optimal patient selection and early prediction of response is an unmet need.

Lymphopenia has been suggested as a negative predictive marker for ICI therapy response in several solid tumors including HNSCC $(4,5)$. Several studies have previously assessed the predictive value of various peripheral lymphocyte measures for ICI therapy response, including absolute or relative lymphocyte count at the beginning of treatment, on-treatment, or dynamic changes during treatment. High baseline ALC and increase in ALC during the treatment were associated with better response to anti-PD-1/PD-L1 therapy $(4,6,7)$. Similarly, low NLR and decrease in NLR with anti-PD-1 blockade were associated with improved outcomes in various solid tumors $(5,8,9)$. The measure of peripheral lymphocyte with the best predictive value in HNSCC is unknown. In this study, we examined comparative predictive values of multiple lymphocyte level measures for anti-PD-1 ICI therapy in patients with advanced HNSCC.

\section{Materials and methods}

Patient selection. We retrospectively collected clinicopathologic data on patients diagnosed with advanced HNSCC who have received at least two doses of anti-PD-1 ICI therapy at 
Massachusetts General Hospital between 2015 and 2020. Patients who had underlying hematologic malignancy, were taking systemic corticosteroids at the time of therapy, were on antibiotic therapy for an active infection, and/or whose laboratory values were not available were excluded from the study.

Variables and follow up. Demographic data, clinicopathologic data, medical and social history, treatment history, and treatment responses were obtained from the patients' records. Baseline and week 6 absolute lymphocyte count (ALC) and absolute neutrophil count (ANC) were collected, and neutrophil-to-lymphocyte ratios (NLR) were calculated. ALC and NLR changes $(\Delta)$ from baseline to week 6 were measured.

Statistical analysis. Progression-free survival (PFS), defined as the time elapsed between the initiation of ICI therapy and tumor progression or death from any cause, was the primary clinical outcome measure. Overall survival (OS) was defined as the time from the initiation of ICI treatment to the date of death from any cause. The Kaplan-Meier method was used to estimate PFS and OS, and the Cox proportional hazards regression model was used to estimate hazard ratios (HR) and confidence intervals (CI). Treatment response was defined as unequivocal radiographic and/or clinical improvement on ICI treatment, and was summarized using exact $95 \%$ CIs for each subgroup. The multivariable models were adjusted for human papilloma virus (HPV)-association, smoking history, Eastern Cooperative Oncology Group (ECOG) performance status, and the line of ICI therapy. All statistical tests were 2-sided, and a $\mathrm{P}$ value of $<0.05$ was considered statistically significant. This study was conducted under the IRB Protocol no. 2018P001456.

\section{Results}

Patient characteristics. Between the years 2015 and 2020, a total of 108 patients with advanced HNSCC that met the selection criteria for this study were identified. The patients' baseline demographic and clinicopathologic characteristics and treatment history are summarized in Table I. The median (range) age was 67 (26-92) years. Eighty-one point five percent of the patients were male and $88.0 \%$ of the patients were white. The oropharynx was the most common primary tumor site (31.5\%) followed by the oral cavity (26.9\%). Anti-PD-1 therapy was given as a first-line therapy in $47.2 \%$ of the patients. Thirty-one (28.7\%) cases were human papilloma virus (HPV) associated (Table I). Median baseline ALC, ANC, and NLR were 790 cells $/ \mu 1$, 4,780 cells $/ \mu 1$, and 6.7 , respectively. Week 6 ALC, ANC, and NLR were 770 cells $/ \mu 1,4,670$ cells $/ \mu 1$, and 6.2 , respectively. Median $\triangle \mathrm{ALC}$ and $\triangle \mathrm{NLR}$ were -15 and 6.1 , respectively.

Survival analysis. With a median follow-up of 11.7 months (range, 1.4-51.1), the median OS (mOS) for the total population was 18.4 months (95\% CI, 14.5-22.3) and the median PFS (mPFS) was 4.1 months (95\% CI, 3.1-5.1). Neither baseline ALC nor NLR provided predictive value for PFS. The mPFS for patients with high $(\geq 790)$ vs. low $(<790)$ baseline ALC were 4.0 months (95\% CI, 3.3-5.5) and 4.0 months (95\% CI, 2.2-5.8), respectively (Fig. 1A). The mPFS for patients with high $(\geq 6.7)$ vs. low $(<6.7)$ baseline NLR were 4.4 months (95\% CI, 3.0-5.8) and 4.1 months (95\% CI, 2.6-5.6), respectively (Fig. 1B). On
Table I. Baseline demographic and clinicopathologic characteristics and treatment history of the total population $(n=108)$.

\begin{tabular}{lr}
\hline Clinicopathologic characteristics & Value \\
\hline Median age, years (range) & $67(29-92)$ \\
Sex, $\mathrm{n}(\%)$ & \\
Male & $88(81.5)$ \\
Female & $20(18.5)$ \\
Ethnicity, $\mathrm{n}(\%)$ & \\
White & $95(88.0)$ \\
Asian & $11(10.2)$ \\
Black & $2(0.2)$ \\
Performance status (ECOG), $(\%)$ & \\
0,1 & $77(71.3)$ \\
$2,3,4$ & $31(28.7)$ \\
Smoking, $\mathrm{n}(\%)$ & \\
$\geq 10$ PY & $53(49.1)$ \\
Never or $<10$ PY & $55(50.9)$ \\
Primary site, $\mathrm{n}(\%)$ & \\
Oropharynx & $34(31.5)$ \\
Oral cavity & $29(26.9)$ \\
Nasopharynx & $17(15.7)$ \\
Larynx/hypopharynx & $15(13.9)$ \\
Sinonasal & $9(8.3)$ \\
Unknown primary & $4(3.7)$ \\
HPV status, $\mathrm{n}(\%)$ & \\
Positive & $31(28.7)$ \\
Unknown/Negative & $71(72.3)$ \\
Treatment history & \\
Anti-PD-1 agent, $\mathrm{n}(\%)$ & \\
Pembrolizumab & \\
Nivolumab & \\
Line of ICI therapy, $\mathrm{n}(\%)$ & $32(35.4)$ \\
First line & \\
$\geq$ Second line & $51(47.2)$ \\
\hline PY, & $57(52.8)$ \\
\hline
\end{tabular}

PY, pack year; HPV, human papilloma virus; PD-1, programmed cell death 1; ICI, immune checkpoint inhibitor; ECOG, Eastern Cooperative Oncology Group.

the other hand, both week 6 ALC and week 6 NLR were predictive for PFS. High week 6 ALC levels were associated with a longer PFS. The mPFS for patients with high $(\geq 770)$ vs. low $(<770)$ levels at week 6 ALC were 5.6 months (95\% CI, 0.2-11.0) and 3.1 months (95\% CI, 2.2-4.1), respectively (HR, 0.51; 95\% CI, 0.33-0.78; P=0.002, Fig. 1C). Low week 6 NLR was also predictive for improved PFS. The mPFS for patients with high $(\geq 6.2)$ vs. low $(<6.2)$ levels at week 6 NLR were 2.9 months (95\% CI, 2.3-3.5) and 8.7 months (95\% CI, 2.7-14.7), respectively (HR, 0.47; 95\% CI; 0.31-0.73, $\mathrm{P}=0.001$, Fig. 1D). Increase in ALC on-treatment at week 6 (positive $\triangle \mathrm{ALC}$ ) was associated with a trend for longer PFS but was not statistically significant (Fig. 1E). Decrease in NLR on-treatment at week 6 (negative $\Delta \mathrm{NLR}$ ) was associated with a 
Table II. PFS of subgroups based on various peripheral lymphocyte values and other clinical variables.

\begin{tabular}{|c|c|c|c|}
\hline Variable & $\begin{array}{c}\text { Median } \\
\text { PFS, months } \\
(95 \% \mathrm{CI})\end{array}$ & HR & P-value \\
\hline Total population & $4.1(3.1-5.1)$ & $\mathrm{N} / \mathrm{A}$ & N/A \\
\hline \multicolumn{4}{|l|}{ ALC values } \\
\hline \multicolumn{4}{|l|}{ Baseline ALC } \\
\hline High $(\geq 790)$ & $4.0(3.3-5.5)$ & 0.87 & 0.521 \\
\hline Low $(<790)$ & $4.0(2.2-5.8)$ & & \\
\hline \multicolumn{4}{|l|}{ Baseline NLR } \\
\hline High $(\geq 6.7)$ & $4.4(3.0-5.8)$ & 1.12 & 0.619 \\
\hline Low $(<6.7)$ & $4.1(2.6-5.6)$ & & \\
\hline \multicolumn{4}{|l|}{ Week 6 ALC } \\
\hline High $(\geq 770)$ & $5.6(0.2-11.0)$ & 0.51 & 0.002 \\
\hline Low $(<770)$ & $3.1(2.2-4.1)$ & & \\
\hline \multicolumn{4}{|l|}{ Week 6 NLR } \\
\hline High $(\geq 6.2)$ & $2.9(2.3-3.50)$ & 0.47 & 0.001 \\
\hline Low $(<6.2)$ & $8.7(2.7-14.7)$ & & \\
\hline \multicolumn{4}{|l|}{$\triangle \mathrm{ALC}$} \\
\hline Positive & $5.1(3.5-6.7)$ & 0.78 & 0.269 \\
\hline Negative & $3.6(2.5-4.7)$ & & \\
\hline \multicolumn{4}{|l|}{$\Delta \mathrm{NLR}$} \\
\hline Positive & $2.7(2.1-3.3)$ & 0.58 & 0.015 \\
\hline Negative & $6.7(2.9-10.5)$ & & \\
\hline \multirow{2}{*}{\multicolumn{4}{|c|}{$\begin{array}{l}\text { Other characteristics } \\
\text { HPV }\end{array}$}} \\
\hline & & & \\
\hline Positive & $4.2(0.7-7.7)$ & 0.90 & 0.660 \\
\hline Unknown/negative & $4.1(3.1-5.1)$ & & \\
\hline \multicolumn{4}{|l|}{ Line of ICI therapy } \\
\hline First line & $5.1(2.7-7.5)$ & 0.76 & 0.216 \\
\hline$\geq$ Second line & $3.8(3.0-4.6)$ & & \\
\hline \multicolumn{4}{|l|}{ ECOG } \\
\hline 0,1 & $4.4(2.6-6.2)$ & 0.69 & 0.117 \\
\hline $2,3,4$ & $3.1(1.4-4.8)$ & & \\
\hline \multicolumn{4}{|l|}{ Smoking } \\
\hline$\geq 10 \mathrm{PY}$ & $3.8(2.9-4.7)$ & 1.17 & 0.461 \\
\hline Never or $<10 \mathrm{PY}$ & $4.7(2.3-7.1)$ & & \\
\hline
\end{tabular}

PFS, progression-free survival; CI, confidence interval; HR, hazard ratio; ALC, absolute lymphocyte count; NLR, neutrophil-to-lymphocyte ratio; $\Delta$, difference; HPV, human papilloma virus; ICI, immune checkpoint inhibitor; ECOG, Eastern Cooperative Oncology Group; PY, pack year.

superior PFS. The mPFS for patients with negative vs. positive $\triangle$ NLR were 6.7 months (95\% CI, 2.9-10.5) and 2.7 months (95\% CI, 2.1-3.3), respectively (HR, 0.58; 95\% CI, 0.38-0.90; $\mathrm{P}=0.015$, Fig. 1F). Other clinicopathologic characteristics, including HPV status, line of ICI therapy, ECOG status, and smoking history, were examined for correlation with disease progression. None of the subgroups showed a differential impact on PFS (Table II). The differential predictive value of week 6 ALC, week 6 NLR, and $\triangle$ NLR for PFS all remained
Table III. Response rate of subgroups based on various peripheral lymphocyte values and other clinical variables.

\begin{tabular}{|c|c|c|}
\hline Variable & Response rate $(95 \% \mathrm{CI})$ & HR \\
\hline Total population & $22.2(14.8-31.2)$ & N/A \\
\hline \multicolumn{3}{|l|}{ ALC values } \\
\hline \multicolumn{3}{|l|}{ Baseline ALC } \\
\hline High $(\geq 790)$ & $24.1(13.5-37.6)$ & 0.645 \\
\hline Low $(<790)$ & $20.4(10.6-33.5)$ & \\
\hline \multicolumn{3}{|l|}{ Baseline NLR } \\
\hline High $(\geq 6.7)$ & $25.5(14.7-39.0)$ & 0.412 \\
\hline Low $(<6.7)$ & $18.9(9.4-32.0)$ & \\
\hline \multicolumn{3}{|l|}{ Week 6 ALC } \\
\hline High $(\geq 770)$ & $24.1(21.1-47.5)$ & 0.645 \\
\hline Low $(<770)$ & $20.4(10.6-33.5)$ & \\
\hline \multicolumn{3}{|l|}{ Week 6 NLR } \\
\hline High $(\geq 6.2)$ & $17.9(8.9-30.4)$ & 0.263 \\
\hline Low $(<6.2)$ & $26.9(15.6-41.0)$ & \\
\hline \multicolumn{3}{|l|}{$\triangle \mathrm{ALC}$} \\
\hline Positive & $20.0(10.0-33.7)$ & 0.611 \\
\hline Negative & $24.1(13.9-37.2)$ & \\
\hline \multicolumn{3}{|l|}{$\Delta \mathrm{NLR}$} \\
\hline Positive & $20.7(11.2-33.4)$ & 0.682 \\
\hline Negative & $24.0(13.1-38.2)$ & \\
\hline \multicolumn{3}{|l|}{ Other characteristics } \\
\hline \multicolumn{3}{|l|}{ HPV } \\
\hline Positive & $22.6(9.6-41.1)$ & 0.955 \\
\hline Unknown/negative & $22.1(13.4-33.0)$ & \\
\hline \multicolumn{3}{|l|}{ Line of ICI therapy } \\
\hline First line & $27.5(15.9-41.7)$ & 0.214 \\
\hline$\geq$ Second line & $17.5(8.7-29.9)$ & \\
\hline \multicolumn{3}{|l|}{ ECOG } \\
\hline 0,1 & $22.1(13.4-33.0)$ & 0.955 \\
\hline $2,3,4$ & $22.6(9.6-41.1)$ & \\
\hline \multicolumn{3}{|l|}{ Smoking } \\
\hline$\geq 10 \mathrm{PY}$ & $22.6(12.3-36.2)$ & 0.921 \\
\hline Never or $<10 \mathrm{PY}$ & $21.8(11.8-35.0)$ & \\
\hline
\end{tabular}

CI, confidence interval; HR, hazard ratio; ALC, absolute lymphocyte count; NLR, neutrophil-to-lymphocyte ratio; $\Delta$, difference; HPV, human papilloma virus; ICI, immune checkpoint inhibitor; ECOG, Eastern Cooperative Oncology Group; PY, pack year.

statistically significant after a multivariable analysis $(\mathrm{P}=0.009$, 0.005 , and 0.037 , respectively).

Response rate analysis. The response rate to anti-PD-1 therapy for the total group of patients with advanced HNSCC was $22.2 \%$ (95\% CI, 14.8-31.2), including three complete responses and 21 partial responses (Table III). Response rates were not significantly different between high vs. low baseline ALC and NLR cohorts, although patients with high ALC and low NLR values at baseline and week 6 had numerically higher response rates. The response rate for patients with high $(\geq 790)$ or low (<790) baseline ALC were 24.1\% (95\% CI, 13.5-37.6) 

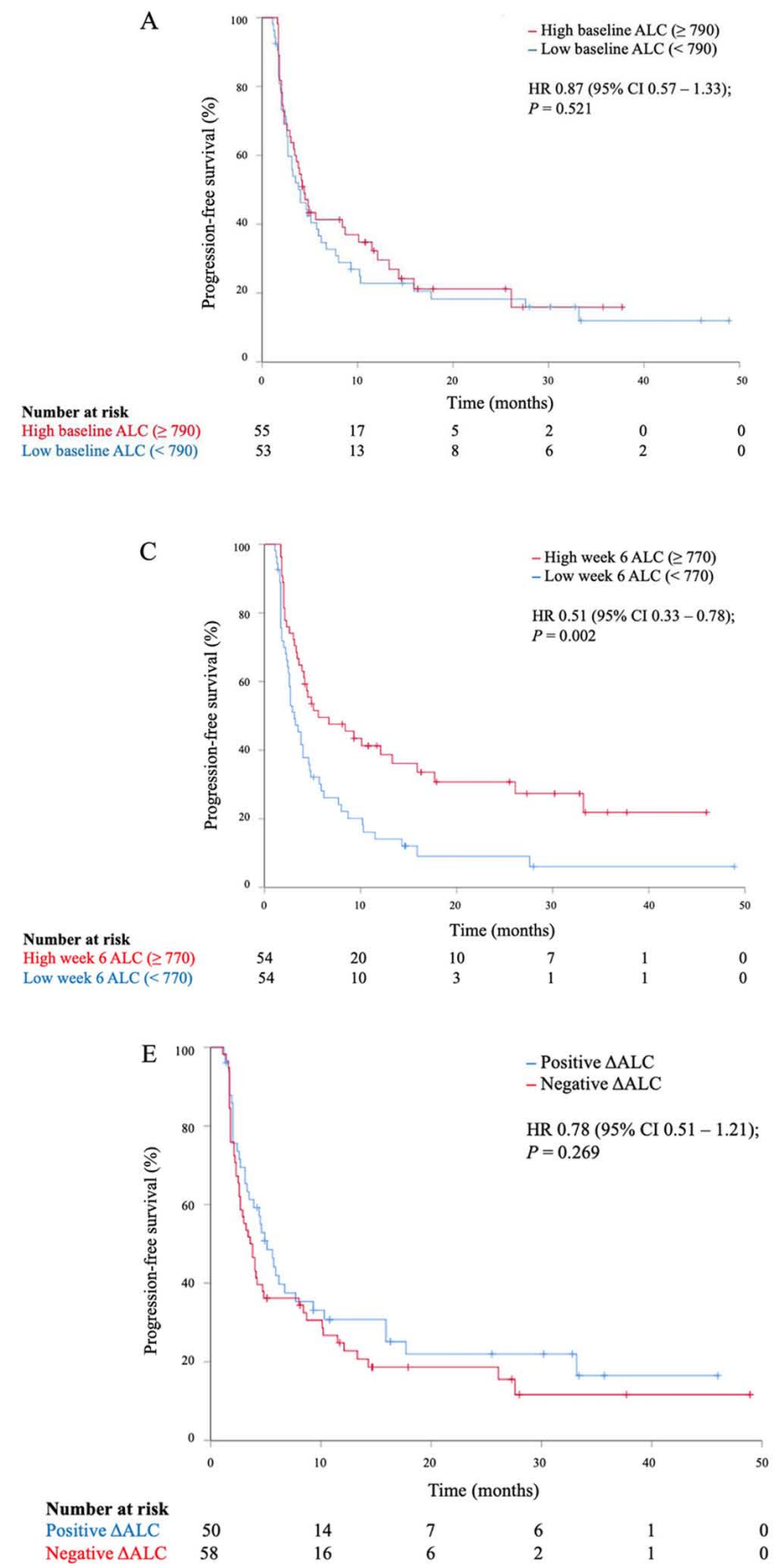

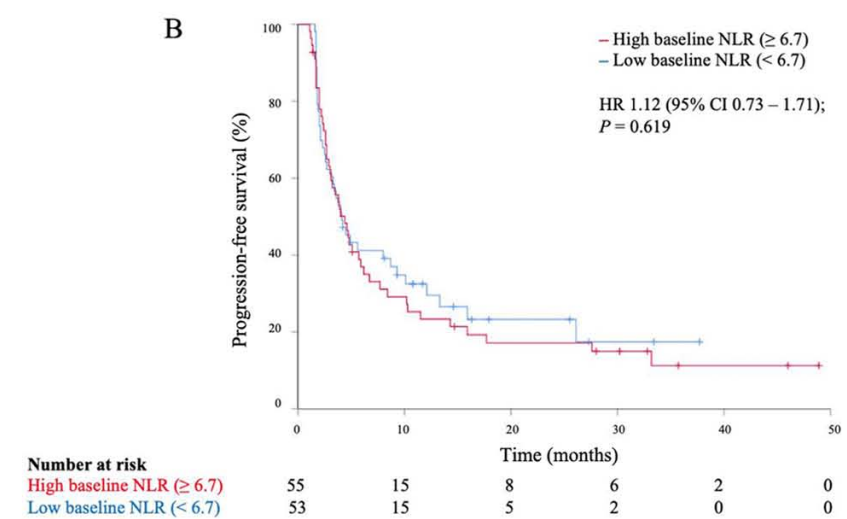

$\mathrm{D}$

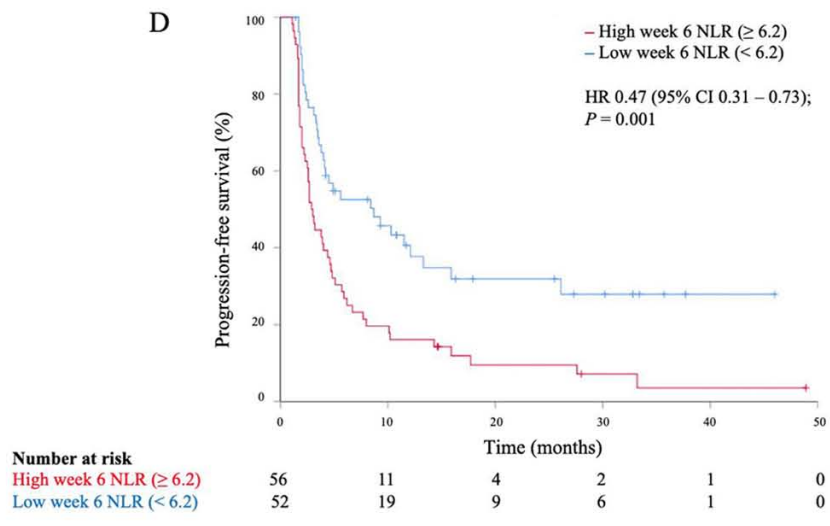
Number at risk
High week 6 NLR $(\geq 6.2)$ Low week 6 NLR $(<6.2)$
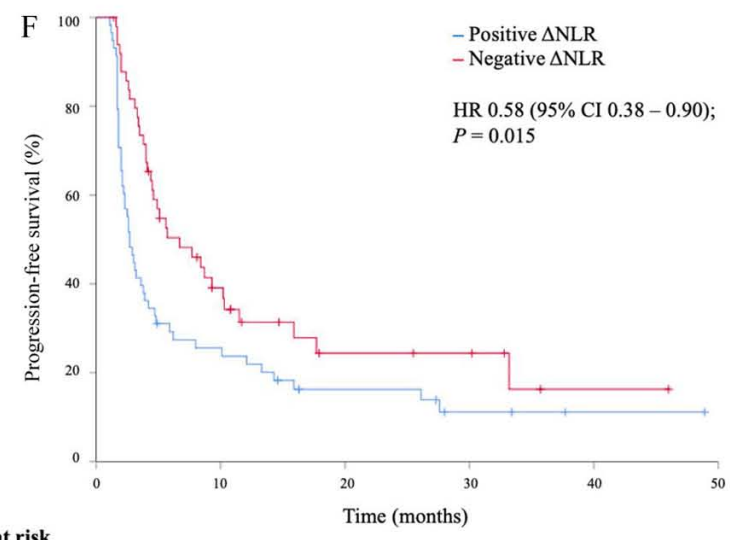

Number at risk

Positive $\triangle N L R$

Negative $\triangle N L R$

Figure 1. Kaplan-Meier progression-free survival curves. Kaplan-Meier progression-free survival curves of patients with HNSCC treated with anti-PD-1 therapy based on various peripheral lymphocyte measures. (A) Baseline ALC. (B) Baseline NLR. (C) Week 6 ALC. (D) Week 6 NLR. (E) $\triangle A L C$. (F) $\triangle N L R$. HNSCC, head and neck squamous cell carcinoma; PD-1, programmed cell death 1; ALC, absolute lymphocyte count; NLR, neutrophil-to-lymphocyte ratio; $\triangle \mathrm{ALC}$, change of absolute lymphocyte count from baseline to week $6 ; \triangle \mathrm{NLR}$, change of neutrophil-to-lymphocyte ratio from baseline to week 6; HR, hazard ratio; $\mathrm{CI}$, confidence interval.

and $20.4 \%$ (95\% CI, 10.6-33.5), and the response rate for patients with high $(\geq 6.7)$ or low $(<6.7)$ baseline NLR were $25.5 \%$ (95\% CI, 14.7-39.0) and $18.9 \%$ (95\% CI, 9.4-32.0), respectively. Similarly, the response rates for patients with high $(\geq 770)$ or low (<770) week 6 ALC were $24.1 \%$ (95\% CI, 21.1-47.5) and $20.4 \%$ (95\% CI, 10.6-33.5), and the response rate for patients with high $(\geq 6.2)$ or low $(<6.2)$ week 6 NLR were $17.9 \%$ (95\% CI, 8.9-7-30.4) and 26.9\% (95\% CI, 15.6-41.0), respectively. On-treatment dynamic changes of ALC and NLR and other baseline clinicopathologic parameters showed no differential effect on response rate (Table III).

\section{Discussion}

In this single institution retrospective study, we examined various measures of peripheral lymphocyte levels in association with response to anti-PD-1 ICI therapy in patients with advanced HNSCC. To our knowledge, this is the first study to explore the correlation of anti-PD-1 immune checkpoint inhibitor therapy with multiple lymphocyte variables in HNSCC. Baseline lymphocyte measures either in absolute level or in proportion with neutrophil count did not predict anti-PD-1 therapy response. However, early on-treatment lymphocyte values for 
both ALC and NLR were significantly associated with ICI treatment outcome. A high week 6 ALC and higher week 6 NLR were associated with longer disease control. A low week 6 NLR was also associated with a trend for higher response rate but was not statistically significant. As per dynamic changes of lymphocyte, decreasing NLR (increasing lymphocyte proportion) change during treatment was associated with a longer PFS but ALC change was not correlated with the outcome.

Several studies previously explored the correlation between peripheral lymphocyte count and ICI therapy outcomes in variable solid tumors. Ho et al (4) reported a correlation between pretreatment $\mathrm{ALC} \geq 600$ cells $/ \mu 1$ and NLR $<7$ with disease control with anti-PD-1 therapy in recurrent or metastatic HNSCC. Similar association between baseline ALC and NLR with ICI therapy outcomes was demonstrated in lung cancer, melanoma, and other solid tumors (5,9-11). In our patient cohort, baseline lymphocyte values had no correlation with ICI outcome. Lalani et al (9) demonstrated that week 6 NLR had a stronger correlation with response rate, PFS, and OS of advanced renal cell carcinoma patients who were treated with ICI therapy, which was more consistent with our findings. In addition, as shown in our HNSCC cohort, the association between NLR changes with ICI outcomes has been observed in various solid tumors $(5,9,12)$.

The major limitation of our study was the inherent bias of retrospective analyses in uncontrolled groups. Despite multivariable analyses, the potential selection bias and the imbalance of the baseline characteristics and treatment history may have contributed to the treatment outcomes. The relatively small sample size may have limited statistical power for subgroup analyses.

In conclusion, our single institution retrospective analysis suggests that week 6 ALC, week 6NLR, and on-treatment NLR dynamic have a predictive value for anti-PD-1 therapy responses. Our findings warrant further investigation of peripheral lymphocyte as a potential clinical marker for ICI therapy in advanced HNSCC.

\section{Acknowledgements}

Not applicable.

\section{Funding}

No funding was received.

\section{Availability of data and materials}

The datasets used and/or analyzed during the current study are available from the corresponding author on reasonable request.

\section{Authors' contributions}

JCP designed the study and wrote the manuscript. JD analyzed the data, created figures and reviewed the manuscript. JRC participated in the study design and reviewed the manuscript. All authors read and approved the final manuscript.

\section{Ethics approval and consent to participate}

The present study was conducted under the IRB Protocol Partners \#2018P001456.

\section{Patient consent for publication}

Not applicable.

\section{Competing interests}

The authors declare that they have no competing interests.

\section{References}

1. Ferris RL, Blumenschein G Jr, Fayette J, Guigay J, Colevas AD, Licitra L, Harrington K, Kasper S, Vokes EE, Even C, et al: Nivolumab for recurrent squamous-cell carcinoma of the head and neck. N Engl J Med 375: 1856-1867, 2016.

2. Burtness B, Harrington KJ, Greil R, Soulières D, Tahara M, de Castro G Jr, Psyrri A, Basté N, Neupane P, Bratland À, et al: Pembrolizumab alone or with chemotherapy versus cetuximab with chemotherapy for recurrent or metastatic squamous cell carcinoma of the head and neck (KEYNOTE-048): A randomised, open-label, phase 3 study. Lancet 394: 1915-1928, 2019.

3. Cohen EEW, Soulieres D, Le Tourneau C, Dinis J, Licitra L, Ahn MJ, Soria A, Machiels JP, Mach N, Mehra R, et al: Pembrolizumab versus methotrexate, docetaxel, or cetuximab for recurrent or metastatic head-and-neck squamous cell carcinoma (KEYNOTE-040): A randomised, open-label, phase 3 study. Lancet 393: 156-167, 2019.

4. Ho WJ, Yarchoan M, Hopkins A, Mehra R, Grossman S and Kang H: Association between pretreatment lymphocyte count and response to PD1 inhibitors in head and neck squamous cell carcinomas. J Immunother Cancer 6: 84, 2018.

5. Ameratunga M, Chénard-Poirier M, Candilejo IM, Pedregal M, Lui A, Dolling D, Aversa C, Garces AI, Ang EJ, Banerji U, et al: Neutrophil-lymphocyte ratio kinetics in patients with advanced solid tumours on phase I trials of PD-1/PD-L1 inhibitors. Eur J Cancer 89: 56-63, 2018.

6. Huemer F, Lang D, Westphal T, Gampenrieder SP, Hutarew G, Weiss L, Hackl H, Lamprecht B, Rinnerthaler G and Greil R: Baseline absolute lymphocyte count and ECOG performance score are associated with survival in advanced non-small cell lung cancer undergoing PD-1/PD-L1 blockade. J Clin Med 8: 1014, 2019.

7. Maltese M, Panni S, Lazzarelli S, Brighenti M, Negri F and Ratti M: High baseline lymphocyte count is a predictive biomarker of prolonged time to progression in patients with advanced solid tumors receiving checkpoint inhibitors. J Clin Oncol 35 (Suppl 15): e14532-e14532, 2017.

8. Zer A, Sung MR, Walia P, Khoja L, Maganti M, Labbe C, Shepherd FA, Bradbury PA, Feld R, Liu G, et al: Correlation of neutrophil to lymphocyte ratio and absolute neutrophil count with outcomes with PD-1 axis inhibitors in patients with advanced non-small-cell lung cancer. Clin Lung Cancer 19: 426-434. e421, 2018.

9. Lalani AKA, Xie W, Martini DJ, Steinharter JA, Norton CK, Krajewski KM, Duquette A, Bossé D, Bellmunt J, Van Allen EM, et al: Change in neutrophil-to-lymphocyte ratio (NLR) in response to immune checkpoint blockade for metastatic renal cell carcinoma. J Immunother Cancer 6: 5, 2018.

10. Bennati C, Mazza V, D'Arcangelo M, Minuti G, Vecchiarelli S, Attilia L, Gili A, Montanari M, Landi L and Cappuzzo F: E5Integrating programmed cell death ligand 1 (PD-L1) and neutrophil to lymphocyte ratio (NLR) as predictive panel of response to nivolumab in non-small cell lung cancer (NSCLC). Ann Oncol 28 (Suppl_6): doi: 10.1093/annonc/mdx426.004.

11. Capone M, Giannarelli D, Mallardo D, Madonna G, Festino L, Grimaldi AM, Vanella V, Simeone E, Paone M, Palmieri G, et al: Baseline neutrophil-to-lymphocyte ratio (NLR) and derived NLR could predict overall survival in patients with advanced melanoma treated with nivolumab. J Immunother Cancer 6: 74, 2018.

12. Moschetta M, Uccello M, Kasenda B, Mak G, McClelland A, Boussios S, Forster M and Arkenau HT: Dynamics of neutrophils-to-lymphocyte ratio predict outcomes of PD-1/PD-L1 blockade. Biomed Res Int 2017: 1506824, 2017. 